DOI: https://doi.org/10.46296/yc.v4i7edespdic2.0084

\title{
COMPRENSIÓN DE LOS 5 SABERES DECLARADOS EN LA UNESCO, DESCRITOS EN LAS COMPETENCIAS GENERALES Y ARTICULADOS EN LAS DESTREZAS CON CRITERIO DE DESEMPEÑO EN EL CURRÍCULUM DEL ECUADOR
}

\section{UNDERSTANDING OF THE 5 KNOWLEDGE DECLARED IN UNESCO, DESCRIBED IN THE GENERAL COMPETENCES AND ARTICULATED IN THE SKILLS WITH PERFORMANCE CRITERIA IN THE ECUADORIAN CURRICULUM}

\author{
Moreira-Chóez Jenniffer Sobeida ${ }^{1 *}$; Huayamave-Jiménez Shirley Verónica²; \\ Zambrano-Alcívar María Viviana ${ }^{3}$ \\ 1, 2, 3 Estudiante de la Maestría de Pedagogía de los Entornos Digitales, Instituto de \\ Posgrado, Universidad Técnica de Manabí, UTM. Portoviejo, Ecuador.
}

*Correo: jenniffer.moreira@utm.edu.ec

\begin{abstract}
Resumen
El aprendizaje basado en los 5 saberes declarados en la UNESCO cumple un papel muy importante en el diseño curricular del sistema educativo ecuatoriano. En consecuencia, el objetivo de esta investigación es analizar la comprensión de los 5 componentes fundamentales para el proceso de enseñanza y aprendizaje que se debe tener en cuenta al abordar las competencias y destrezas con criterio de desempeño como el propósito de destacar su importancia en el diseño curricular del Ecuador. El presente trabajo se realizó con un enfoque analítico documental, tomando como referencias bibliográficas de varios autores. Para dar continuidad al esquema metodológico de esta investigación, se realizó una búsqueda de los factores que influyen en el desarrollo integral del currículo ecuatoriano, donde se plasma propuestas de organismos Internacionales como la UNESCO, OCDE, CRES entre otros. Dicho proceso tuvo como base el diseño investigativo fenomenológico que partió identificar el problema de investigación que permitió desarrollar el análisis de los factores que afectan al curriculum.
\end{abstract}

Palabras claves: Saberes; competencias generales; UNESCO; desempeño; curriculum.

\begin{abstract}
Learning based on the 5 knowledge declared in UNESCO plays a very important role in the curricular design of the Ecuadorian educational system. Consequently, the objective of this research is to analyze the understanding of the 5 fundamental components for the teaching and learning process that must be taken into account when addressing the competences and skills with performance criteria as the purpose of highlighting their importance in design curriculum of Ecuador. The present work was carried out with a documentary analytical approach, taking as bibliographic references of several authors. To give continuity to the methodological scheme of this research, a search was carried out for the factors that influence the integral development of the Ecuadorian curriculum, where proposals from international organizations such as UNESCO, OECD, CRES, among others, are reflected. This process was based on the phenomenological research design that began to identify the research problem that allowed to develop the analysis of the factors that affect the curriculum.
\end{abstract}

Keywords: Knowledge; general skills; UNESCO; performance; resume.

Información del manuscrito:

Fecha de recepción: 01 de diciembre de 2020.

Fecha de aceptación: 30 de noviembre de 2020.

Fecha de publicación: 31 de diciembre de 2020. 


\section{Introducción}

Las Instituciones de educación se la define como una práctica social de enseñar saberes y conocimientos a los estudiantes. "El aprendizaje es aprendizaje del saber y de los conocimientos. Se trata del tiempo, en el cual el saber y los conocimientos se hacen aprendizaje, es decir producción articulada y coherente de sentidos" (Cullen, 1997). Por lo tanto, estos elementos centrales de la educación (enseñanza y aprendizaje) se pueden transmitir siempre y cuando se vinculan al sujeto con el objeto, por medio de conocimiento, saberes, habilidades, entre otros.

Es por eso por lo que, es necesaria la existencia del vínculo entre el profesor, estudiante y saber, cuyos complementos contribuyen en el crecimiento de cada persona y de su entorno, involucrando los conocimientos que requieren estar presentes en la institución sean estos semejantes o diversos, de manera que permitan aprehender de la realidad.

Por lo tanto, el currículo es esencial en la práctica experimental y formativa del maestro siendo este un implemento que sustenta y contribuye en el proceso de enseñanza y aprendizaje tanto en las clases presencial como virtuales, a fin de poder emplear una secuencia multidireccional que permite al estudiante crecer en una formación integral desarrollando habilidades y destrezas para su crecimiento personal y profesional.

Dentro de este marco, es necesario un análisis de los problemas que afectan el desarrollo curricular en las instituciones educativas, pero para ello, es importante conocer las bases que sustentan la educación y su articulación en el currículum nacional.

\section{Metodología}

Este trabajo investigativo es de carácter metodológico a través de una investigación analítica documental, tomando como base referencias bibliográficas de varios autores. Por ende, el objetivo de este trabajo es analizar la comprensión de los 5 componentes fundamentales para el proceso de enseñanza y aprendizaje que se debe tener en cuenta al abordar las competencias y destrezas con 
criterio de desempeño como el

propósito de destacar su importancia en el diseño curricular del Ecuador, donde se podrá plasmar si la aplicación de estos componentes de enseñanza y aprendizaje, contribuye al cumplimiento eficiente de las diversas actividades académicas ligadas a la misión, visión y objetivos de la del sistema educativo ecuatoriano.

\section{Desarrollo}

La Organización de las Naciones Unidas para la Educación, la Ciencia y la Cultura (UNESCO) en sus declaraciones nos dice que la "educación es un derecho humano para todos, a lo largo de toda la vida, y que el acceso a la instrucción debe ir acompañado de la calidad". En los últimos 50 años y mediante diferentes publicaciones, plantea 5 saberes que los considera como competencias generales útiles para la humanidad.

Figura 1. Los 5 Saberes de la Educación.

\begin{tabular}{|c|c|c|}
\hline $\begin{array}{l}\text { Saber (APRENDER A } \\
\text { CONOCER) } \\
\text { - Cómo aprende el } \\
\text { cerebro } \\
\text { - Tipos de } \\
\text { aprendizaje } \\
\text { - Estilo de } \\
\text { aprendizaje }\end{array}$ & & $\begin{array}{l}\text { Saber CONVIVIR } \\
\text { - Empatía } \\
\text { - Acuerdo } \\
\text { - Principios de la Gestalt } \\
\text { - Programación } \\
\text { Neurolingüística } \\
\text { - Hábitos de la gente } \\
\text { altamente afectiva }\end{array}$ \\
\hline $\begin{array}{l}\text { Saber HACER } \\
\text { - Fases del proceso creativo } \\
\text { - Facilitadores y obstáculo } \\
\text { de la creatividad } \\
\text { - Creatividad individual y } \\
\text { grupal }\end{array}$ & $\begin{array}{l}\text { Saber SER } \\
\text { - Emociones } \\
\text { - Valores y Creencias } \\
\text { - Autoestima } \\
\text { - Autorrealización } \\
\text { - Autoconocimiento }\end{array}$ & $\begin{array}{l}\text { Saber TRASCENDER } \\
\text { - Acción aislada y conjunta } \\
\text { - Desarrollar habilidades } \\
\text { - Estilo de vida } \\
\text { - Promover el conocimiento } \\
\text { y prácticas } \\
\text { - Actuar para lograr la } \\
\text { solidaridad social. }\end{array}$ \\
\hline
\end{tabular}

Fuente: Elaboración propia a partir de la UNESCO

Los saberes son de vital importancia en nuestras vidas los cuales contribuyen en el desarrollo de las actividades que realizamos cada día.
A continuación, se describen brevemente cada uno de ellos.

Saber (Conocer), se lo puede definir como el que aprende de manera autónoma, combinando el 
aprendizaje personal como el de su entorno. Este elemento de aprendizaje se centra en la atención, memoria y en la adquisición de conocimientos ya agrupados y clasificados.

Aprender a hacer, se enfoca en la práctica y experiencia de lo aprendido; es decir, mediante la realización de diversas tareas como tangible e intangible.

Saber Ser, se orienta a la personalidad del Ser, sus características, sus emociones y lo que necesita para desarrollar al máximo sus capacidades y habilidades.

Saber vivir juntos, es aprender a vivir con grupos de individuos relacionados con su entorno, donde conlleva un aprendizaje significativo en base al descubrimiento de los problemas y en busca de soluciones para así fomentar el conocimiento ante los demás.

Saber trascender, es la necesidad del ser humano de buscar nuevos desafíos, cada acción realizada sea aislada o conjunta contribuyen en el aprendizaje del individuo.

Por consiguiente, los conceptos de los saberes se encuentran vinculados con las competencias, toda vez que existen dos fases que los complementan tanto como objeto educativo y como componente de planeación didáctica y se define claramente cómo se enlazan los dos elementos.

\section{La Educación}

\section{¿Cómo interiorizamos estos saberes en la educación?}

Para responder a esta interrogante necesitamos conocer varios conceptos que nos ayuden a entender lo que significa la educación para la sociedad.

(Pestalozzi, 1928) nos dice que "La educación es el desarrollo natural, progresivo y sistemático de todas las facultades".

Mientras que Spencer lo ve como "La función de educar es el proceso de preparar al hombre para la vida completa".

\section{¿Cómo podemos definir a la educación?}

Tomando como base estos conceptos, podemos decir que la educación es un proceso de preparar al hombre para el desarrollo de su vida, la convivencia y la transformación de la sociedad. 
Para conocer sobre la educación en

Ecuador es importante remitirnos a documentos oficiales que enmarcan claramente la misión de la educación en nuestro país, por esto se toma como referencia a la Constitución del Ecuador 2008 en Art. 27, que nos dice:

"La educación se centrará en el ser humano y garantizará su desarrollo holístico, en el marco del respeto a los derechos humanos, al medio ambiente sustentable y a la democracia; será participativa, obligatoria, intercultural, democrática, incluyente y diversa, de calidad y calidez; impulsará la equidad de género, la justicia, la solidaridad y la paz; estimulará el sentido crítico, el arte y la cultura física, la iniciativa individual y comunitaria, y el desarrollo de competencias y capacidades para crear y trabajar" (Registro Oficial $N^{\circ}$ 503, 2019)

Haciendo un análisis sobre nuestros conceptos oficiales decimos que la educación es un derecho de todos, debe ser inclusiva en todos sus niveles con el fin de formar ciudadanos reflexivos, auténticos y competentes para desarrollar una mejor sociedad.
Por ello, uno de los retos que tiene las Instituciones de Educación es cumplir con la necesidad de formar estudiantes competitivos y con capacidades esenciales, que les permitan desenvolverse en este mundo globalizado, donde los conocimientos adquiridos se consolidarán con la práctica y experiencia, permitiendo el desarrollo de las habilidades y capacidades cognitivas.

Para cumplir con el ideal de la educación necesitamos herramientas que guíen el propósito. Cada localidad o país enfoca sus objetivos con diferentes lineamientos que ayuden a comprender mejor este camino.

\section{El Currículum}

El principal instrumento que marca el inicio es el currículo. Conocido como un proyecto que organiza las actividades educativas, los objetivos, contenidos y evaluaciones, diseñado en función del desarrollo social y tecnológico, se lo puede definir como un contrato social cuyo objetivo primordial es la transformación de la personalidad del individuo que se aspira formar para su desarrollo personal y profesional. 
(González, 1994) define al currículo como un proyecto estructurado de formación y un proceso sistemático de contenidos y experiencias de aprendizaje, con propuesta político educativa que propugnan diversos sectores sociales, a fin de producir aprendizajes significativos que se interprete en formas de pensar, sentir, valorar y actuar frente a los problemas complejos que plantea la vida social y laboral.

El Curriculum del Ecuador tiene como objetivo primordial la formación integral de los estudiantes, alcanzar el máximo desempeño como seres humanos capaces, creativos, humanísticos, culturales, artísticos, deportivos, donde el docente fomenta y organiza actividades complementarias a los planes de estudio, lo definimos como un Currículum Psicologista Dialéctico con enfoque en competencias.

\section{Niveles de Concreciones Curricular}

Por su parte, en el Art. 9 del Acuerdo Ministerial N. ㅇ ME-2015-00168-A, señala que con el objetivo de que el ejercicio de planificación curricular cumpla la meta de atención a la diversidad, considerada en el marco legal educativo, se requiere una distribución de responsabilidades en el desarrollo del diseño curricular que comprenda tres niveles de concreción (Ministerio de Educación, 2015).

1. Primer nivel: Ministerio de Educación, a cargo de la planificación macro curricular (currículo nacional obligatorio);

2. Segundo nivel: Instituciones educativas, a cargo de la planificación meso curricular; corresponde al currículo de la institución educativa, en articulación con el currículo nacional; está plasmada en la Proyecto Curricular Institucional $(\mathrm{PCl})$ y la Planificación Curricular Anual (PCA); se articula e incluye en el PEl; responde a las especificidades y el contexto de cada institución y a la pertinencia cultural propia de los pueblos $y$ nacionalidades indígenas, y

3. Tercer nivel: Docentes, a cargo de la planificación micro curricular y que corresponde al currículo del aula e incluye las adaptaciones curriculares precisas para la atención de necesidades educativas especiales; esta se elabora basándose en la $\mathrm{PCl}$ y en correspondencia con la PCA. 
Desde otra perspectiva, el Macrocurrículo es el primer nivel de planificación curricular liderado por el Ministerio de Educación y un conjunto de expertos en varios campos del conocimiento como: investigadores, científicos, especialistas, docentes entre otros. En este punto se plasman objetivos, campo de estudio, perfil de ingreso, perfil de egreso y otros componentes que comprenden la realidad inmediata y las necesidades de la comunidad y estudiantes, apoyado en las bases y disposiciones legales como: Leyes, Decretos, Reglamentos e Instrucciones para el ámbito autónomo.

El segundo nivel de concreción está conformado por el Meso currículo. En esta estructura curricular se determinan dos programas, la Planificación Curricular Institucional
(PCl) y la Planificación Curricular Anual (PCA), las cuales son diseñadas por los directivos $y$ docentes de las entidades, adaptadas a las necesidades curriculares en función de la institución.

El Micro currículo, es la etapa final del desarrollo del proceso de enseñanza aprendizaje, he aquí donde el docente desarrolla las planificaciones, PEA (Planificación Estratégica por Asignatura) y Syllabus como parte de su responsabilidad académica, en la cual debe responder a los principios del modelo educativo Institucional, garantizando de manera teórica y práctica los criterios pedagógicos y cuyos protagonistas son los docentes y estudiantes en el proceso dinámico e interactivo de enseñanza y aprendizaje.

Tabla 1. Niveles de Concreción Curricular.

\begin{tabular}{|c|c|c|}
\hline 1 Nivel Macro Currículo & 20 Nivel Meso Currículo & 3 Nivel Micro Currículo \\
\hline Ministerio de Educación & Instituciones Educativas & Docentes \\
\hline Currículo Nacional Obligatorio & $\begin{array}{l}\text { Currículo Institucional } \\
\text { aProyecto Curricular } \\
\text { Institucional } \\
\square \text { Plan Curricular Anual }\end{array}$ & $\begin{array}{l}\text { Currículo de aula } \\
\square \text { Planificaciones de aula } \\
\square \text { Adaptaciones curriculares } \\
\square \text { (Individuales y grupales) }\end{array}$ \\
\hline Prescriptivo & Flexible & Flexible \\
\hline
\end{tabular}

Fuente: Elaborado por (Ministerio de Educación, 2016). Obtenido de: https://educacion.gob.ec/wp-content/uploads/downloads/2016/03/planificacionescurriculares.pdf 


\section{Fundamentos del Diseño Curricular}

Según lo expuesto por (González, 1994) los fundamentos del currículo es un sistema teórico y metodológico referencial, integrado por diferentes disciplinas científicas, que sustentan una propuesta y orientan el análisis de las condiciones socioeconómicas y la construcción del modelo curricular. Por consiguiente, citando a (Silverio Gómez, 2005) sostiene que los fundamentos curriculares se detallan en todo el Currículo, de manera explícita o implícita, pero siempre presentes; de no ser así no cumplirían con los propósitos y objetivos, que es orientar todo el proceso teórico, constructivo, práctico y evaluativo del Currículo.

Para fundamentar las orientaciones del diseño curricular se debe determinar los objetivos de la educación, considerando una serie de interrogantes que permitan orientar los métodos, procedimientos, técnicas $\mathrm{y}$ estrategias, para garantizar el nivel de enseñanza de las IES, tales como ¿qué enseñar?, ¿cómo enseñar?, ¿cuándo enseñar? y ¿qué, ¿cómo y cuándo evaluar? En este sentido, se permitirá planificar de forma global y secuencial las diversas actividades académicas de acuerdo con los planes operativos y Syllabus.

(Bolaños Bolaños \& Molina Bogantes, 1990), señalan que el alumno es un sujeto de experiencias de aprendizaje, por lo tanto, el docente debe estimular la responsabilidad para que el estudiante realice su propio proceso de aprendizaje.

Para aquello, se debe emplear actividades autónomas que permitan el desarrollo de sus habilidades sin asistencia del maestro. Por otra parte, el docente debe asumir el rol de guía o facilitador del proceso de aprendizaje, para aquello debe ser creativo y dinámico utilizando técnicas y recursos que permitan el aprendizaje independiente.

\section{Dimensiones del Diseño} Curricular

El (Ministerio de Educación, 2016) sostiene que los elementos principales del Currículo para el Sistema Educativo Ecuatoriano van en concordancia con cada nivel y subnivel de enseñanza o educación bilingüe intercultural. 
Figura 2. Dimensiones del Diseño Curricular.

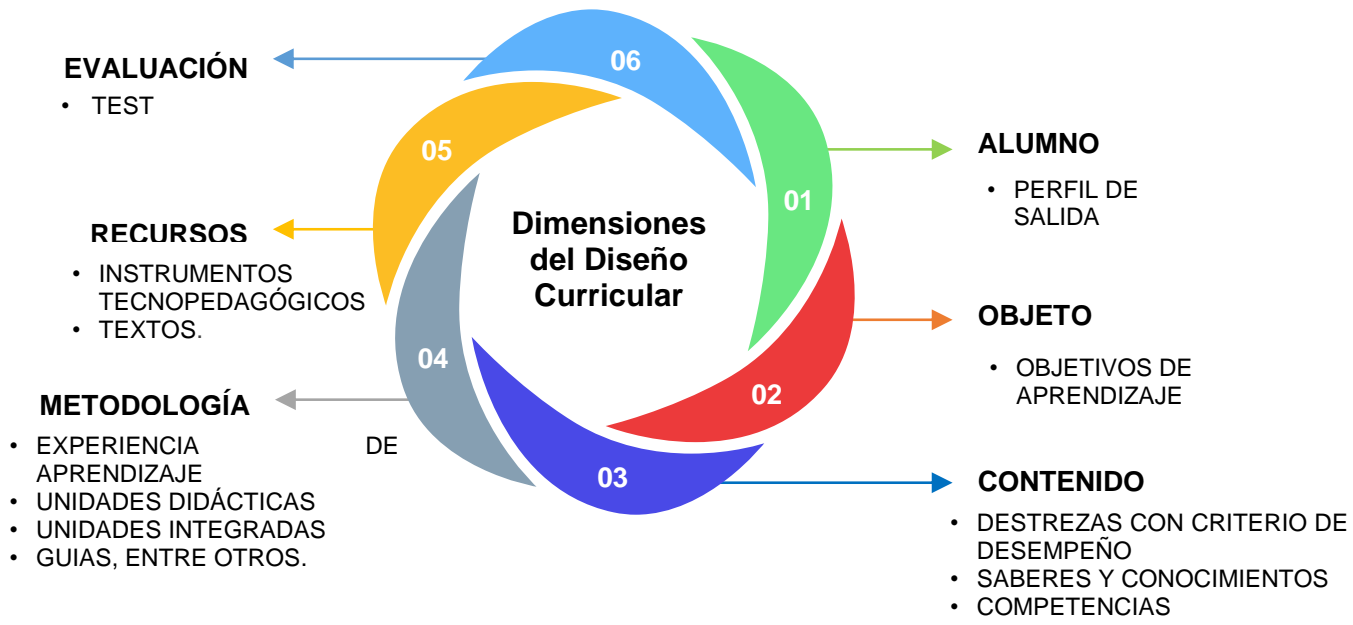

Fuente: Elaboración propia a partir del (Ministerio de Educación, 2016). Obtenido de: https://educacion.gob.ec/wp-content/uploads/downloads/2016/03/planificacionescurriculares.pdf

El Ministerio de Educación ha desarrollado los curriculum de Educación General Básica y de Bachillerato General Unificado partiendo desde el perfil de salida que espera lograr, este es el primer y principal elemento del Curriculum dado que es el centro de la propia organización. Una vez declarado el perfil de salida podemos describir los objetivos de aprendizaje, que han sido dados por cada subnivel tales como: Básica Superior, Básica Media, Básica Elemental y Básica Preparatoria.

Los contenidos han sido organizados por bloques curriculares, en los que se especifica las destrezas con criterio de desempeño por área y por subnivel dejando así claramente escrito lo que se debe enseñar.
Se fomenta una metodología basada en la actividad y la participación del estudiante, se propone el diseño de tareas que motiven al estudiante partiendo de situaciones reales, que contemplen los ritmos y estilos de aprendizaje de cada estudiante para así favorecer el aprendizaje por sí mismo y en equipos de trabajo.

Las Instituciones educativas disponen de autonomía pedagógica y organizativa para el desarrollo del currículum y el logro de sus objetivos, lo que debe ser estructurado por nivel, subnivel y área del conocimiento.

El ministerio brinda recomendaciones y criterios de evaluación propuestos en el currículo, haciendo énfasis en las actividades de evaluación formativa. 
Considerando la autonomía que se da a las Instituciones, cada una es capaz de ofertar algún área adicional a la descrita en el presente currículum, así como objetivos, contenidos y metodologías de acuerdo a su proyecto educativo institucional.

\section{Áreas del conocimiento del Diseño Curricular}

El Curriculum del Ecuador tanto para la Educación Básica General como para el Bachillerato General Unificado se encuentra organizado por las siguientes áreas del conocimiento.

Tabla 2. Áreas del Conocimiento.

\begin{tabular}{|c|c|c|}
\hline Áreas de Conocimiento & Asignatura para EGB & Asignatura para BGU \\
\hline Lengua y Literatura & Lengua y Literatura & Lengua y Literatura \\
\hline Lengua Extranjera & Inglés & Inglés \\
\hline Matemática & Matemática & Matemática \\
\hline \multirow{2}{*}{ Ciencias Naturales } & Ciencias Naturales & Química \\
\cline { 3 - 3 } & & Biología \\
\cline { 3 - 3 } Ciencias Sociales & \multirow{2}{*}{ Estudios Sociales } & Física \\
\cline { 3 - 3 } & & $\begin{array}{c}\text { Eductoria } \\
\text { Educación para la } \\
\text { ciudadanía }\end{array}$ \\
\hline Educación Cultural y Artística & Educación Cultural y & Educación Física \\
\hline Interdisciplinaridad & Artística & Educación Cultural y Artística \\
\hline
\end{tabular}

Fuente: Elaborado por (Ministerio de educación, 2016). Obtenido de: https://educacion.gob.ec/wp-content/uploads/downloads/2016/03/Curriculo1.pdf

Luego de conocer el recorrido que hacen los Saberes (UNESCO) para articularse en las áreas de conocimiento es importante evaluar el resultado del sistema educativo en el Ecuador para conocer si logramos la meta propuesta, y es allí, donde nos encontramos con problemáticas que crecen día a día sin lograr observar una pronta solución.
Problemáticas y causas que afectan al sistema educativo

El Ministerio de Educación (MINEDUC, 2018) identificó problemas en el sector educativo, de la cual se espera la disminución de la problemática a través de las soluciones regulatorias planteadas a fin de garantizar el avance $y$ desarrollo de esta entidad 
gubernamental. Cuyos problemas se detallan a continuación:

1. Deserción estudiantil

2. Ingreso limitado de los grupos de atención prioritaria a la educación

3. Bajo rendimiento académico en los estudiantes

4. Deficiencia en la gestión de procesos educativos complementarios

5. Exceso de estudiantes en las Instituciones Educativas

6. Pérdida de la Identidad cultural y lingüística en las instituciones educativas con Educación Intercultural Bilingüe

7. Vulneración de Derechos a docentes y estudiantes

Las causas que han generado estas problemáticas se dan por diversos factores que son más críticos en el sector público, a continuación, mencionamos las más relevantes:

- Falta de atención a la diversidad desde el currículo.

* Falta de socialización de los documentos de meso planificación (Son las planificaciones curriculares más amplias, Planificación Curricular Institucional $\mathrm{PCl}$ y Planificación Curricular Anual PCA son el referente curricular de la Institución Educativa y de éstas, los docentes tienen los lineamientos para planificar sus clases).

* Inadecuada aplicación del currículo de idiomas.

- Evaluaciones estandarizadas no ajustadas a la medición de logros que contienen los estándares de aprendizaje.

Enseñanza descontextualizada

Las malas condiciones de trabajo docente

Recursos didácticos no adaptados para personas con discapacidades

* El docente limita la implementación de innovaciones, pone barreras al mejoramiento pedagógico digital y al uso de recursos técnicos y tecnológicos que afianzan la calidad, entre otros.

Docentes que no conocen de pedagogía y didáctica. 
Estas problemáticas que se detectan en los niveles de Educación General Básica y Bachillerato General Unificado afectan indudablemente al desarrollo de la sociedad, si agrupamos estas causas en 2, podemos ir en búsqueda de las respuestas que necesitamos.

La causa más grave es la deserción escolar a todo nivel educativo, esto incrementa la pobreza, la falta de pensamiento crítico, de desarrollo creativo y el desempleo.

La segunda y de igual importancia, es que no estamos preparando una generación competente, que pueda hacerse cargo de un futuro que demandará otras habilidades para su supervivencia, esto representa un grave riesgo para la sociedad del siglo $\mathrm{XXI}$, donde la implementación de nuevos saberes entre ellos la tecnología son indispensable para un desarrollo económico.

Con estas realidades que cada día se hacen más notorias y que se evidencian en los jóvenes y en el crecimiento desmedido de la población y de la pobreza, nos brinda la oportunidad de iniciar un proceso de reflexión profunda y nos lleva a tomar acciones inmediatas que ayuden de una $u$ otra forma a corregir este camino a una sociedad en decadencia.

Como miembros de una comunidad educativa, tenemos la obligación de aportar propuestas a mejoras desde nuestros propios espacios.

¿Cuál sería el aporte de la Educación Superior en este camino al cambio?

Para conocer como la Educación Superior puede aportar al cambio de una sociedad es importante conocer sus bases y fundamentos, iniciamos con la Constitución en el Art. 350, señala que el Sistema de Educación Superior tiene como finalidad la formación académica y profesional con visión científica y humanista; la investigación científica y tecnológica; la innovación, promoción, desarrollo y difusión de los saberes y las culturas; la construcción de soluciones para los problemas del país, en relación con los objetivos del régimen de desarrollo (Registro Oficial № 503, 2019).

Es entendido entonces, que el sistema de Educación Superior se debe enfocar en la formación integral del ser humano y entregar a la sociedad ciudadanos con pensamiento crítico capaces de 
desarrollar un estilo de vida sustentable y en armonía con la naturaleza, que puedan generar avances científicos y tecnológicos en beneficio de toda la comunidad.

Las instituciones de educación superior tratan de innovar su currículo para que esté acorde a las nuevas exigencias de su contexto y los diferentes ámbitos de la formación académica de sus docentes facilitando las herramientas que ayuden a la aplicación de la pedagogía y didáctica moderna.

La educación superior está atravesando un proceso de cambio permanente con la inserción de la tecnología la cual ha tomado importancia en el proceso de enseñanza aprendizaje, el nivel académico exige un cambio con metodologías para fortalecer las debilidades encontradas $y$ desarrollar destrezas y aptitudes tanto en docentes como estudiantes.

Podemos definir al currículo de educación superior como una construcción social y colectiva, sustentada en etapas o procesos consecutivos de investigación y evaluación de las tendencias de los saberes, la sociedad, la profesión y de los actores que intervienen en la educación. Determina la visión de la educación y promueve un plan de acción que especifica el proyecto y formación pedagógica, orientado en crear experiencias de aprendizaje significativo en base a la realidad, que inciden en la personalidad y entorno social.

De acuerdo con la Organización para la Cooperación y el Desarrollo Económicos (OCDE) hoy en día es muy importante la participación de la educación superior. Como resultado de las innovaciones digitales y tecnológicas se proporciona un gran valor a las competencias avanzadas, ya que en la actualidad los empleados menos competentes están siendo suprimidos del mundo laboral. Las diversas dificultades que se encuentran en la educación y en el mercado laboral se derivan de las diferencias en los resultados socioeconómicos y en el bienestar general que se transmiten de padres a hijos.

\section{Dimensiones del Currículo} Universitario

Las dimensiones del Currículo de la Educación Superior de acuerdo con el (CES) se desprenden en base a su conceptualización, las cuales se detallan en la siguiente tabla: 
Tabla 3. Dimensiones del Currículo de Educación Superior.

\begin{tabular}{|c|c|l|}
\hline № & Dimensión & \multicolumn{1}{|c|}{ Descripción } \\
\hline $\mathbf{1}$ & Epistemológica & $\begin{array}{l}\text { Referida a la organización de los saberes científicos } \\
\text { estructurados en los campos de estudio del currículo. }\end{array}$ \\
\hline $\mathbf{2}$ & Política & $\begin{array}{l}\text { Relativa a la dinámica del poder expresada en los discursos } \\
\text { pedagógicos y en la intencionalidad de la práctica curricular. }\end{array}$ \\
\hline $\mathbf{3}$ & Educativa & $\begin{array}{l}\text { Para la construcción de redes semánticas (de significado) y } \\
\text { sintácticas (de integración y de sentidos relacionados a la } \\
\text { profesión) del conocimiento. }\end{array}$ \\
\hline $\mathbf{4}$ & Cultural & $\begin{array}{l}\text { Como construcción colectiva de prácticas transformacionales } \\
\text { de los ciudadanos interculturales que pretende formar. }\end{array}$ \\
\hline $\mathbf{5}$ & Investigativa & $\begin{array}{l}\text { Posibilitan la exploración y organización del conocimiento, su } \\
\text { reorientación en función de los contextos y de los objetivos de } \\
\text { cambio e innovación }\end{array}$ \\
\hline $\mathbf{6}$ & Evaluativa & $\begin{array}{l}\text { Como retroalimentación permanente encaminada hacia la } \\
\text { búsqueda de la calidad y la pertinencia. }\end{array}$ \\
\hline
\end{tabular}

Fuente: Elaboración propia a partir del (CES)

La dimensión del currículo se orienta al diseño, estructura del currículo, el desarrollo de los contenidos académicos y la secuencia de la transmisión de los conocimientos. Se divide en varias dimensiones con la finalidad de ayudar a comprender el objetivo de la Planificación Curricular; así mismo, se complementa con los aprendizajes extracurriculares como prácticas experimentales, proyectos, pasantías, entre otros.

La III Conferencia Regional de Educación Superior en América Latina y el Caribe (CRES, 2018) conjuntamente con la UNESCO, han creado un Plan de acción para el desarrollo sostenible de la educación Superior, con el propósito de contribuir con el fortalecimiento de los sistemas educativos, a fin de generar espacios de investigación e innovación para el desarrollo humano, la convivencia democrática y la ciudadanía, sobre los principios de tolerancia, solidaridad y compromiso social. por ello, plantearon 7 ejes temáticos que a continuación se detallan:

1. Educación Superior como parte del sistema educativo en América Latina y el Caribe.

2. Educación superior, diversidad cultural e interculturalidad en América Latina.

3. Educación superior, internacionalización e integración en América Latina y el Caribe. 
4. Rol de la educación superior de cara a los desafíos sociales de América Latina y del Caribe.

5. Investigación científica y tecnológica, e innovación como motores del desarrollo humano, social y económico para América Latina y el Caribe.

6. Papel estratégico de la educación superior en el desarrollo sostenible de América Latina y del Caribe.
7. A cien años de la Reforma de Córdoba. Hacia un nuevo manifiesto de la educación superior latinoamericana.

A continuación, se detalla el plan de acción CRES 2018, cuyo proceso de elaboración fue pensado en todas las etapas, acciones y estratégicas, su estructura está realizada en base a los lineamientos definido por la UNESCO y adaptado para la organización del Marco de Acción Educación 2030.

Tabla 4. Plan de Acción.

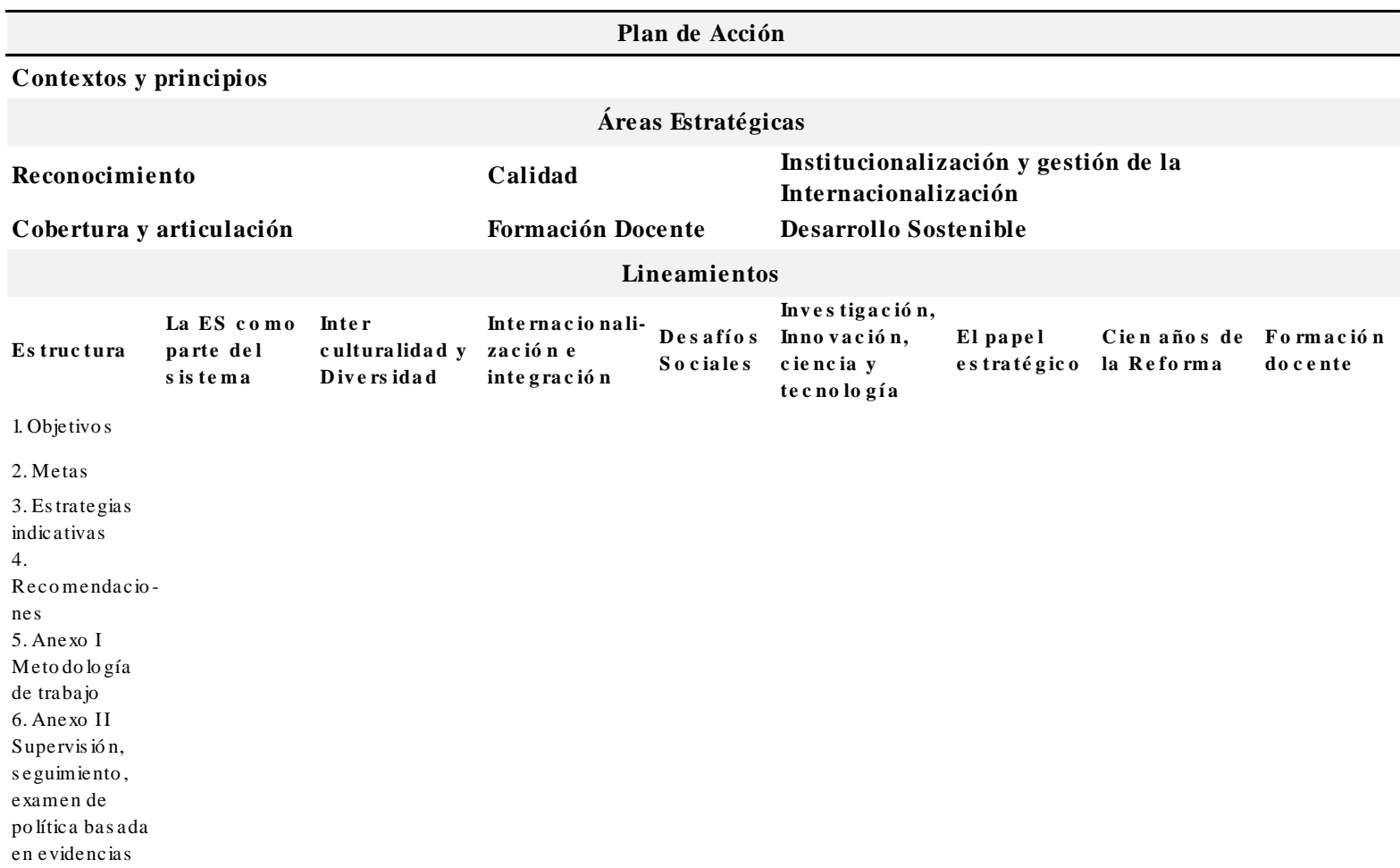

Fuente: Elaborado por (CRES, 2018). Obtenido de: http://www.iesalc.unesco.org/

Por otra parte, la III conferencia Regional de Educación Superior para América Latina y el Caribe, plantea varias recomendaciones para los organismos Internacionales como, continuar reforzando el sistema de formación docente como eje estratégico en los currículos para 
favorecer la integración ética y moral de los futuros docentes.

Así mismo, el (CRES, 2018) exponen varias recomendaciones para las Instituciones de Educación Superior como:

- Reconocer las actividades y proyectos de extensión como parte de los currículos educativos $y$ de la responsabilidad y el compromiso social de la IES.

- Profundizar el perfil internacional del currículo, en particular mediante las actividades de internacionalización en casa (dimensión internacional del currículo, organización de programas académicos colaborativos internacionales tales como dobles títulos, cotutelas, movilidad virtual y convenios institucionales, a nivel nacional e internacional, para el reconocimiento de trayectos formativos).

Por otra parte, la (OCDE) sostiene que es necesario redoblar los esfuerzos para mejorar la equidad en la educación. Por ello, para que exista equidad en la educación los países deben enfocarse en la financiación y la dotación de recursos para la enseñanza de los más vulnerables, evitar la deserción estudiantil, prevenir las pérdidas de curso y fomentar el acceso a la educación de los más pobres. Por consiguiente, es de vital importancia incentivar y contribuir continuamente en la formación y capacitación de los docentes, para la adquisición de nuevos conocimientos pedagógicos que permitan identificar y apoyar a estudiantes de todos los niveles.

\section{Conclusiones}

La finalidad de este trabajo investigativo es realizar un análisis y dar respuesta a las diferentes inquietudes que se presentan en relación con la comprensión de los 5 saberes declarados en la UNESCO, descritos en las competencias generales y articulados en las destrezas con criterio de desempeño en el currículum del Ecuador, los mismos que son considerados como elementos que deben ir ligados en el currículo del sistema educativo.

Es importante conocer cómo influye el currículo en la educación y cómo esto afecta o beneficia al desarrollo económico y social de un país. Si todos los involucrados en los 
procesos de educación tuvieran claros los 5 saberes a los que la educación responde, fuera mucho más fácil articular los objetivos, contenidos, metodologías, recursos y evaluaciones por cada nivel.

Si hacemos un análisis área por área, revisamos y comprendemos los objetivos, los articulamos con los contenidos y los implementamos con metodologías prácticas y reales a nuestra sociedad, el proceso educativo se convertiría en una transformación integral, preocupada por el desarrollo del ser humano y su felicidad. Así mismo, es importante fortalecer el rol de cada miembro de la comunidad educativa y su responsabilidad en el accionar, tomar en consideración la constitución, leyes, reglamentos, normas establecidas por los entes rectores del país, para el desarrollo de la educación.

La Educación a nivel superior se ve en la responsabilidad de actuar ante las habilidades y competencias no desarrolladas a lo largo del proceso educativo con el fin de equilibrar y mejorar el perfil de salida de los estudiantes, el cambio de esquema asignado a la educación superior, como un ente de mero desarrollo de competencias específicas para una profesión, a la responsabilidad de la transformación del individuo como ente de una sociedad activa, emprendedora y llena de buenas personas, ayudará a disminuir la violencia, la mala práctica profesional a cualquier nivel y por ende la pobreza.

Debemos apoyarnos en las recomendaciones planteadas por los organismos internacionales como la UNESCO, OCDE, CRES, entre otros, que permiten viabilizar el desarrollo de la educación, quienes han propuesto un plan de acción conformado por objetivos, estrategias indicativas $y$ recomendaciones, proyecto realizado en base a estudios efectuados por científicos, estadistas, educadores y entre otros miembros de la UNESCO y organizaciones aliadas.

\section{Bibliografía}

Bolaños Bolaños, G., \& Molina Bogantes, Z. (1990). Introducción al Currículo (Primera edición ed.). Universidad Estatal a Distancia, San José (EUNED). Obtenido de 
https://books.google.com.gt/ books?id=Ew_JkA-

5EaUC\&printsec=frontcover\# $v=$ onepage $\& q \& f=$ false

CES. (s.f.). Gestión Curricular. Obtenido de https://www.ces.gob.ec/doc/r egimen_academico/2da_ron da_de_talleres/Mesa3/gestin _curricular_completa.pdf

CRES. (2018). Plan de Acción 2018 - 2028. Obtenido de https://www.iesalc.unesco.or g/2019/02/22/plan-deaccion-cres-2018-2028/

Cullen. (1997). Critica de Las Razones de Educar. Obtenido de https://fdocuments.ec/docum ent/cullen-1997-critica-delas-razones-de-educar.html

González, O. (1994). Diseño Curricular. Obtenido de https://www.academia.edu/3 0977007/Dise\%C3\%B10_cur ricular_Otmara

MINEDUC. (2018). Agenda Regulatoria 2018-2021. Obtenido de https://educacion.gob.ec/wpcontent/uploads/downloads/2 018/06/Agenda-RegulatoriaMineduc-2018.pdf

Ministerio de Educación. (2015). ACUERDO Nro. MINEDUCME-2015-00168-A. Obtenido de

https://educacion.gob.ec/wpcontent/uploads/downloads/2
015/12/ACUERDO-Nro.MINEDUC-ME-201500168.pdf

Ministerio de educación. (2016). Currículo de los niveles de Educación Obligatoria. Obtenido de www.educacion.gob.ec: https://educacion.gob.ec/wpcontent/uploads/downloads/2 016/03/Curriculo1.pdf

Ministerio de Educación. (2016). Instructivo para Planificaciones Curriculares para el Sistema Nacional de Educación.

SUBSECRETARÍA DE FUNDAMENTOS

EDUCATIVOS. Obtenido de https://educacion.gob.ec/wpcontent/uploads/downloads/2 016/03/planificacionescurriculares.pdf

OCDE. (s.f.). Obtenido de https://www.oecd.org/centrod emexico/medios/laocdesosti enequeesnecesarioredoblarl osesfuerzosparamejorarlaeq uidadenlaeducacion.htm

Pestalozzi, J. (1928). Pestalozzi y la nueva Educación. Obtenido de

http://www.bnm.me.gov.ar/gi ga1/documentos/EL003789. pdf

Registro Oficial № 503. (2019). Registro Oficial № 503Constitución de la República del Ecuador. Quito. Obtenido de www.registrofi cial.gob.ec: 
https://www.registroficial.gob .ec/index.php/registro-oficialweb/publicaciones/suplemen tos/item/11713-suplementoal-registro-oficial-no-503

Reyes Añorve, J., \& Godínez Alarcón, G. (2017). Las Tic en la educación superior, innovaciones y retos. Revista Iberoamericana de las Ciencias Sociales y Humanas, Vol. 6(Núm. 12), 18. Las Tic en la educación superior, innovaciones y retos / The ICT in higher education, innovations and challenges:

https://www.ricsh.org.mx/ind ex.php/RICSH/article/view/1 35

Silverio Gómez, M. (2005). Bases y Fundamentos Curriculares. Obtenido de http://www.faacz.com.br/revi staeletronica/links/edicoes/2 005_01/edutec_curriculo_me rcedes_2005_1.pdf

UNESCO. (s.f.). La educación transforma vidas. Obtenido de https://es.unesco.org: https://es.unesco.org/themes /education\#: :text=La\%20U NESCO\%20considera\%20qu e\%20la,los\%20aspectos\%20 de\%20la\%20educaci\%C3\%B $3 n$. 